\title{
Use of transdermal and intravenous granisetron and the ability of the Hesketh score to assess nausea and vomiting induced by multiday chemotherapy
}

\author{
Ralph V Boccia' \\ Gemma Clark ${ }^{2}$ \\ Julian D Howell ${ }^{2}$ \\ 'Center for Cancer and Blood \\ Disorders, Bethesda, MD, USA; \\ 2ProStrakan Pharmaceuticals, \\ Galashiels, UK
}

\author{
This article was published in the following Dove Press journal: \\ Cancer Management and Research \\ 2 July 2012 \\ Number of times this article has been viewed
}

Correspondence: Ralph $\vee$ Boccia Center for Cancer and Blood Disorders, 6420 Rockledge Dr, No 4100,

Bethesda, MD 20817, USA

$\mathrm{Tel}+|30157| 0019$

Fax + I 301 5710988

Email rboccia@ccbdmd.com
Purpose: Hesketh scores define emetogenicity of single-agent and multiagent single-day chemotherapy. This analysis determined the emetogenicity of multiagent, multiday chemotherapy and the Granisetron Transdermal System (GTDS; Sancuso ${ }^{\circledR}$ ).

Methods: This was a retrospective analysis of a multicenter, randomized, double-blind, phase III noninferiority trial of GTDS versus oral granisetron in patients receiving 3 days of multiagent moderately or highly emetogenic chemotherapy, regardless of granisetron formulation. Emesis was defined as vomiting/retching or the use of rescue medication. Logistic regression and classification trees were used to determine the optimal combination of Hesketh scores over the multiagent, multiday regimens for the prediction of emesis.

Results: Of 393 patients, 272 (69.2\%) were chemotherapy naïve. The most common types of cancer were lung (30.5\%) and gynecologic $(21.9 \%)$. The most common chemotherapeutic regimen (in $14.2 \%$ of patients) was cisplatin plus etoposide on days $1-3$. The best binary emesis predictor was day 1 Hesketh score. Patients with a day 1 Hesketh score of 5 had the highest rate of emesis $(62.5 \%)$ versus patients with a score $<5(31.7 \%)$. For patients with day 1 Hesketh score $<5$, only $14.3 \%$ of those receiving only one drug on day 1 experienced emesis.

Conclusion: Hesketh emetogenicity scores of individual agents are applicable to multiday, multiagent chemotherapeutic regimens in patients receiving antiemetics.

Keywords: chemotherapy-induced nausea and vomiting, emetogenicity, granisetron, clinical trial, retrospective analysis

\section{Introduction}

The prevention of chemotherapy-induced nausea and vomiting (CINV) ranks among the most challenging supportive care issues in oncology. Since the development by Hesketh et $\mathrm{al}^{1}$ of a classification and scoring system for the acute emetogenicity of chemotherapeutic agents over 15 years ago, several well-developed classifications of emetogenicity for single doses of individual chemotherapeutic agents have become available. ${ }^{2-4}$ However, the emetogenic potential of combination and multiday chemotherapeutic regimens is poorly defined. Multiday regimens are more common with oral antineoplastic agents (both cytotoxic and biologic), and the emetogenicity of these regimens may become apparent only after several days of continuous administration. ${ }^{2}$ Moreover, the acute and delayed phases of CINV overlap, and the optimal dosage regimens of antiemetic agents when this occurs are not well defined. ${ }^{2,4,5}$ 
The challenge of preventing CINV during multiday regimens prompted the development of a new formulation of the 5-HT $\mathrm{HT}_{3}$ receptor antagonist granisetron: the Granisetron Transdermal System (GTDS; Sancuso ${ }^{\circledR}$, ProStrakan Pharmaceuticals, Galashiels, UK). The GTDS patch is designed to provide continuous delivery of granisetron through the skin over 7 days, with an exposure similar to that of a $2 \mathrm{mg}$ /day oral dose. ${ }^{6}$ A recently published phase III trial in 641 patients receiving their first cycle of a new multiday moderately or highly emetogenic chemotherapeutic regimen demonstrated the noninferiority of GTDS compared with oral granisetron in the primary end point of complete CINV control (60\% vs $65 \%$, respectively; difference $-5 \%$ [95\% confidence interval $-13 \%,+3 \%]){ }^{7}$ We conducted a retrospective analysis of data from this phase III trial to assess existing methods of determining emetogenicity of 3-day chemotherapeutic regimens in patients receiving granisetron, to identify predictors of emetogenicity, and to devise rules for evaluating the emetogenicity of 3-day chemotherapeutic regimens.

\section{Material and methods Data source}

Data were obtained exclusively from a randomized, doubleblind, phase III clinical trial conducted at 60 centers in the US, Europe, India, and Mexico in $2006{ }^{7}$ The primary objective of the trial was to demonstrate noninferiority of the efficacy of GTDS compared with that of oral granisetron. The efficacy and safety of GTDS and oral granisetron were compared during 3- to 5-day administration of moderately or highly emetogenic chemotherapeutic regimens. Antiemetic efficacy was evaluated starting from the first administration of chemotherapy and continuing until 24 hours after the last administration. Granisetron was administered either as a transdermal patch $24-48$ hours before the first dose of chemotherapy or as an oral capsule 1 hour before each day's administration. ${ }^{7}$ Patients used diaries to record the presence and severity of nausea on a 4-point scale (none, mild, moderate, severe) and the presence and severity of vomiting on a 5-point scale (none, mild, moderate, severe, very severe). ${ }^{7}$

Antiemetics such as neurokinin NK1 receptor antagonists, dopamine receptor antagonists, and other $5-\mathrm{HT}_{3}$ receptor antagonists were prohibited, except as rescue medication according to each site's standard of care. Use of corticosteroids as a rescue medication (rather than part of the chemotherapy regimen) was recorded.

This current retrospective analysis comprised data from a subset of 393 patients who had received exactly 3 days
Table I Demographics and baseline characteristics of patients in a phase III trial receiving their first cycle of a new multiday moderately or highly emetogenic regimen $(n=393)$

\begin{tabular}{ll}
\hline Characteristic & Value \\
\hline Age, years, mean (SD) & $55.9(12.0)$ \\
Women, \% & 57.0 \\
No alcohol consumption, \% & 72.3 \\
Smoking status, \% & \\
Never smoked & 55.5 \\
Ex-smoker & 28.0 \\
Smoker & 16.5 \\
Chemotherapy naive, \% & 69.2 \\
Type of cancer, \% & \\
Lung & 30.5 \\
Gynecologic & 21.9 \\
Breast & 15.5 \\
Gastrointestinal & 8.7 \\
Head and neck & 6.6 \\
Lymphoma & 6.1 \\
Other & 10.7 \\
\hline
\end{tabular}

of chemotherapy and either formulation of granisetron; the 3-day subset was the largest in the study and was therefore selected for the analysis. For this analysis, GTDS and oral granisetron were assumed to provide equivalent prophylaxis against nausea and vomiting, so data from both treatment groups were included.

\section{Statistical analysis}

In this analysis, the end point for each patient was emesis, defined as the experience of vomiting or retching or the use of rescue medication during the period from the first administration of chemotherapy until 24 hours after the last

Table 2 Chemotherapy regimens administered to patients in a phase III trial receiving their first cycle of a new multiday moderately or highly emetogenic regimen $(n=393)$

\begin{tabular}{ll}
\hline Chemotherapy regimen & $\begin{array}{l}\text { Percentage } \\
\text { of patients }\end{array}$ \\
\hline Cisplatin/etoposide days I-3 & 14.2 \\
Cisplatin/5-FU days I-3 & 10.4 \\
Cisplatin/gemcitabine day I, cisplatin days 2-3 & 9.7 \\
Cisplatin/vinorelbine day I, cisplatin days 2-3 & 9.4 \\
Cisplatin days I-3 & 4.6 \\
Cisplatin/cyclophosphamide day I, cisplatin days 2-3 & 4. I \\
Doxorubicin/cyclophosphamide day I-3 & 3.8 \\
Fludarabine/cyclophosphamide day I-3 & 3.6 \\
5-FU/epirubicin/cyclophosphamide days I-2, & 2.8 \\
5-FU/cyclophosphamide day 3 & \\
5-FU/epirubicin/cyclophosphamide days I-2, & 2.5 \\
epirubicin/cyclophosphamide day 3 & \\
Other (frequency < 10) & 34.9 \\
\hline
\end{tabular}

Abbreviation: 5-FU, 5-fluorouracil. 
administration (ie, not achieving a complete response to granisetron).

Emetogenicity of the multiday regimens was based on the emetogenic potential of individual chemotherapeutic agents as defined by Hesketh et al. ${ }^{1}$ In this system, agents are classified according to proportion of patients expected to experience emesis with each agent in the absence of effective antiemetic prophylaxis as follows: level $1,<10 \%$ of patients; level 2 , $10 \%-30 \%$ of patients; level $3,30 \%-60 \%$ of patients; level 4 , $60 \%-90 \%$ of patients; and level $5,>90 \%$ of patients. ${ }^{1}$

The Hesketh score was determined for each agent on each of the 3 days of chemotherapy. The three overall daily

\section{A}

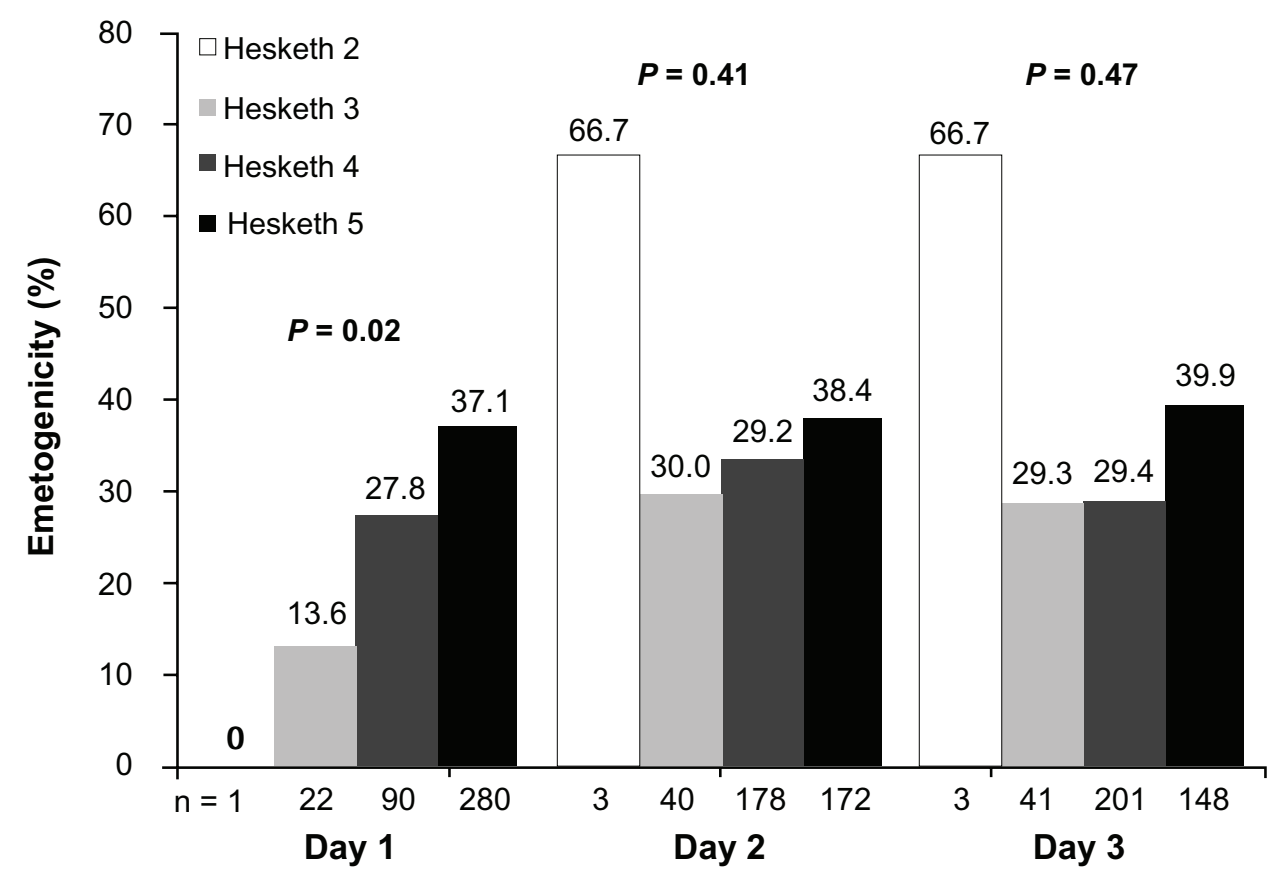

B

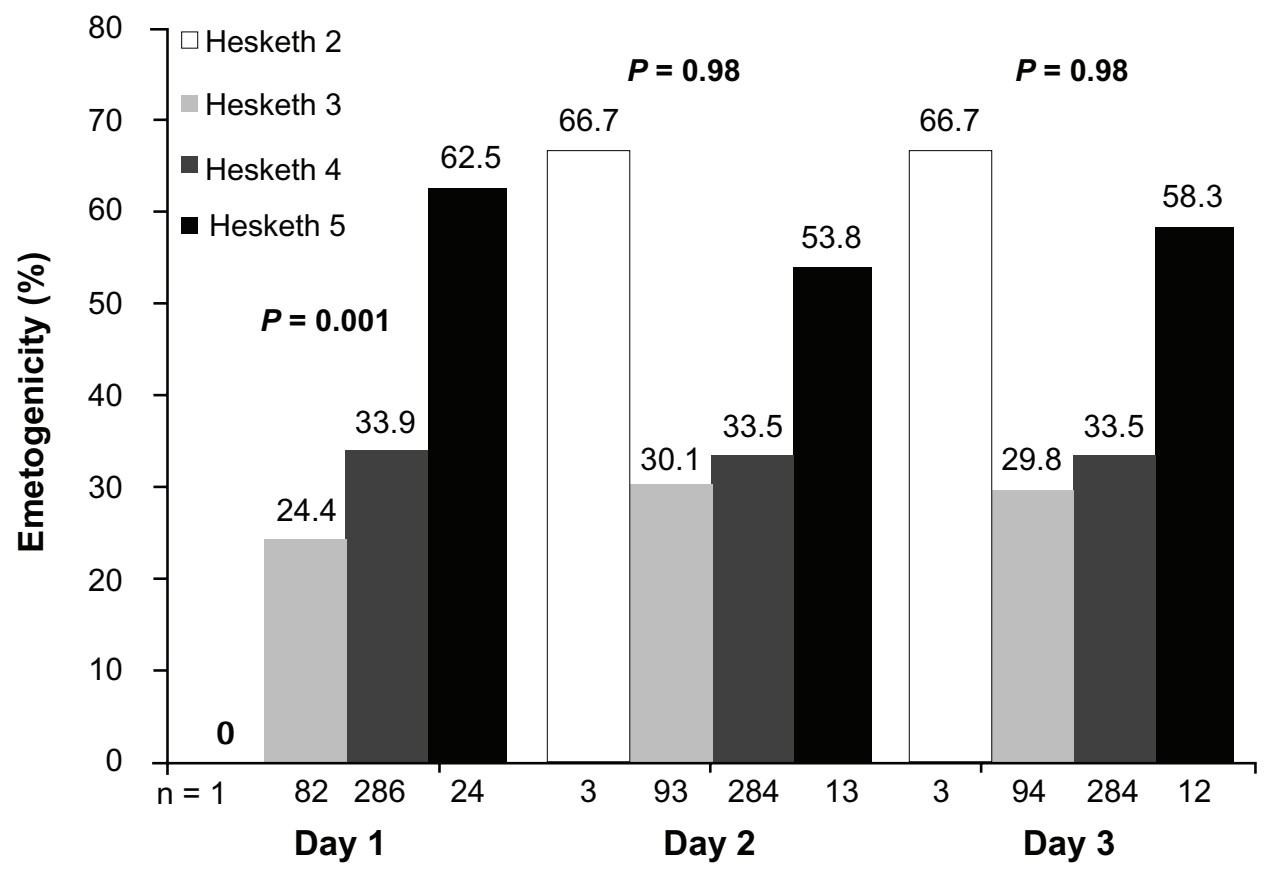

Figure I Emetogenicity (percentage of patients expected to experience emesis) of multiday, multiagent chemotherapy regimens by overall Hesketh scores' of 2, 3, 4, or 5 on each day of chemotherapy (scores determined by the emetogenic potential of each chemotherapeutic agent administered each day). 
scores were then calculated using the algorithm defined by Hesketh et $\mathrm{al}^{1}$ by first identifying the agent with the highest emetogenicity level and then adding to the score according to the emetogenicity levels of the remaining agents. This score was calculated as follows: no increase for the addition of a level 1 agent, a 1-point increase for the addition of one or more level 2 agents (regardless of the number of agents), and a 1-point increase for each level 3 or 4 agent. ${ }^{1}$ The overall emetogenicity of the multiagent, multiday, chemotherapeutic regimen was then defined as the maximum of the three individual daily scores.

The utility of this predefined overall emetogenicity score for multiday regimens was explored by tabulating the rates of emesis for each overall score.

The relationships between the emetogenicity of the chemotherapy regimen and reported emesis, as well as other patient characteristics, were further explored using both logistic regression analysis and classification trees. The classification tree approach uses a statistical technique called recursive partitioning. At each step, factors are examined to determine which ones best separate patients who experienced emesis from those who did not. All analyses were conducted using the statistical software $\mathrm{R}$ version 2.12.0, Institute for Science and Mathematics (Vienna, Austria). Patient characteristics explored were age, sex, smoking status, alcohol consumption, and chemotherapy naïvety. Emetogenicity of the multiday regimen was characterized using the scores of the most emetogenic and the second, third, and fourth most emetogenic agents on each of days 1, 2, and 3, as well as the three daily scores.

\section{Results}

The current analysis comprised 393 patients who received exactly 3 days of chemotherapy and either the transdermal or oral formulation of granisetron. Patient demographics and baseline characteristics are shown in Table 1. The mean age of the population was 55.9 years, $57.0 \%$ were women, and $69.2 \%$ were chemotherapy naïve. The most common malignancies were lung (30.5\%), gynecologic (21.9\%), and breast $(15.5 \%)$ cancers.

Patients were treated with a range of chemotherapeutic regimens (Table 2). The three most common regimens were daily cisplatin plus etoposide in 56 patients $(14.2 \%)$, daily cisplatin plus fluorouracil in 41 patients $(10.4 \%)$, and daily cisplatin plus gemcitabine (day 1 only) in 38 patients $(9.7 \%$ ).

The frequency of emesis increased with increasing Hesketh score. For patients with a score of 3, 4, and 5, the proportion who experienced emesis was $10 \%, 29 \%$, and $37 \%$, respectively. The reported frequency of emesis was

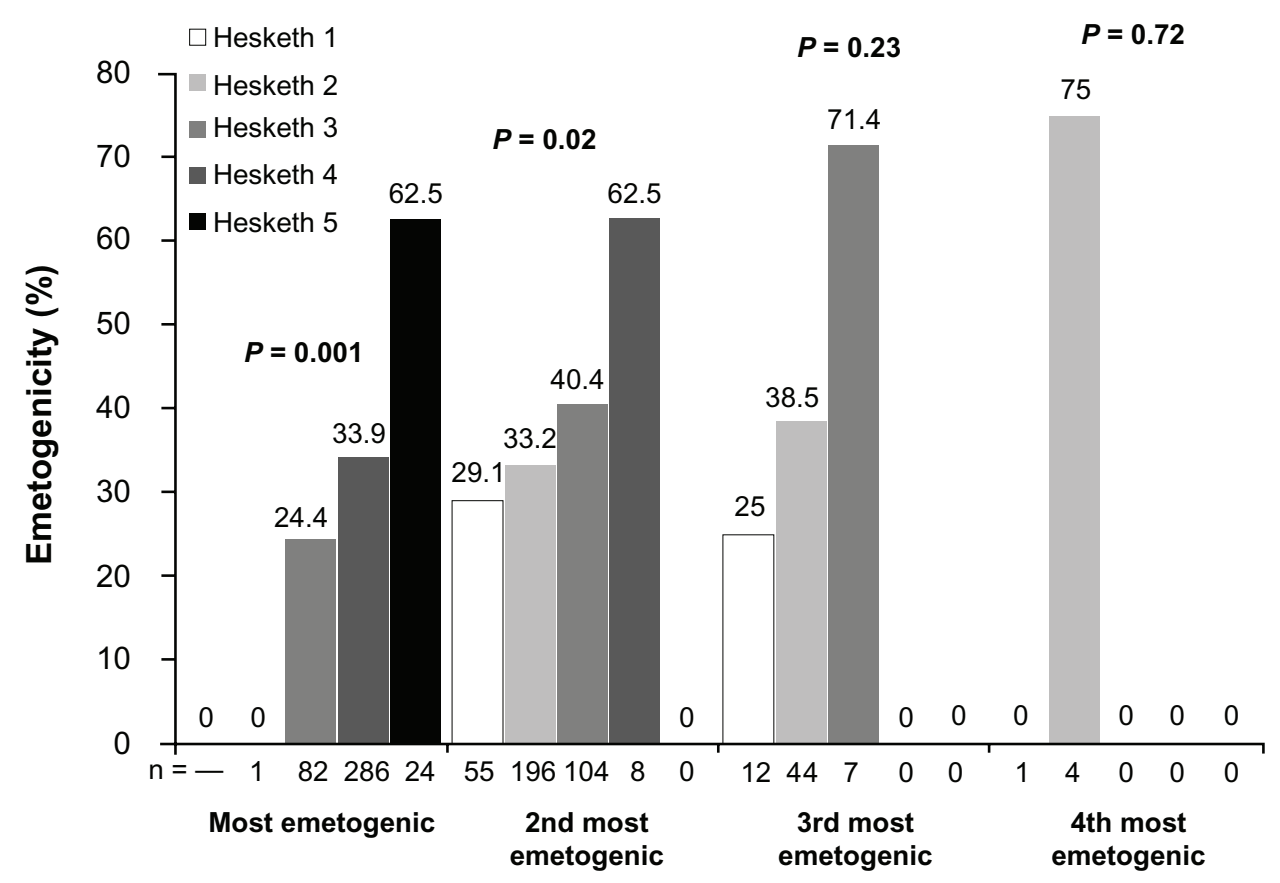

Figure 2 Emetogenicity (percentage of patients expected to experience emesis) of the four most emetogenic multiday, multiagent chemotherapy regimens by individual Hesketh scores' of I, 2, 3, 4, or 5 on day I of chemotherapy (logistic regression analysis). Hesketh scores determined by the emetogenic potential of each chemotherapeutic agent administered on day $\mathrm{I}$. 
lower than predicted by the calculated Hesketh score, but this was not unexpected, given that the original study was based on patients receiving no antiemetic treatment and all patients in the current subanalysis received prophylactic granisetron. For the three most common chemotherapeutic regimens, the overall emetogenicity score was 5 . The original Hesketh algorithm predicted emesis would have been $90 \%$ for a score of 5 and $60 \%-90 \%$ for a score of 4 . However, the observed emesis for the three regimens was 34\% (cisplatin plus etoposide), 39\% (cisplatin plus fluorouracil), and 24\% (daily cisplatin plus gemcitabine).

Use of corticosteroids as a rescue medication (rather than part of a chemotherapy regimen) was recorded. For patients on a planned 3-day chemotherapy regimen, there were no consistent differences in the percentage of patients requiring rescue medication between the GTDS and the oral groups (34/202 [16.8\%] vs 28/201 [13.9\%] during the primary end point evaluation period). For patients on a planned 4/5-day regimen, the percentage of patients given rescue medication was higher in the GTDS group than in the oral group (28/106 [26.4\%] vs $11 / 112$ [9.8\%] during the primary end point evaluation period), but the number of evaluable patients was relatively small.

\section{Logistic regression analysis}

Using logistic regression, the overall Hesketh score on day 1 was found to be a significant predictor of emesis $(P=0.02)$, with scores on subsequent days of the multiday regimen providing no further information regarding emetogenicity (Figure 1). When considering individual agents given on day 1 , the agent with the highest score $(P=0.001)$ and the agent with the second highest score $(P=0.02)$ were found to predict emetogenicity for the regimen (Figure 2).

\section{Classification tree analysis}

Using the classification tree analysis, the best binary predictor of emetogenicity over the period at risk was the most emetogenic agent given on day 1. Patients whose maximum Hesketh score on day 1 was 5 had the highest rate of emesis $(62.5 \%)$; those whose maximum score on day 1 was 4 or less and who had only one drug on day 1 had the lowest rate of emesis (14.3\%) (Figure 3).

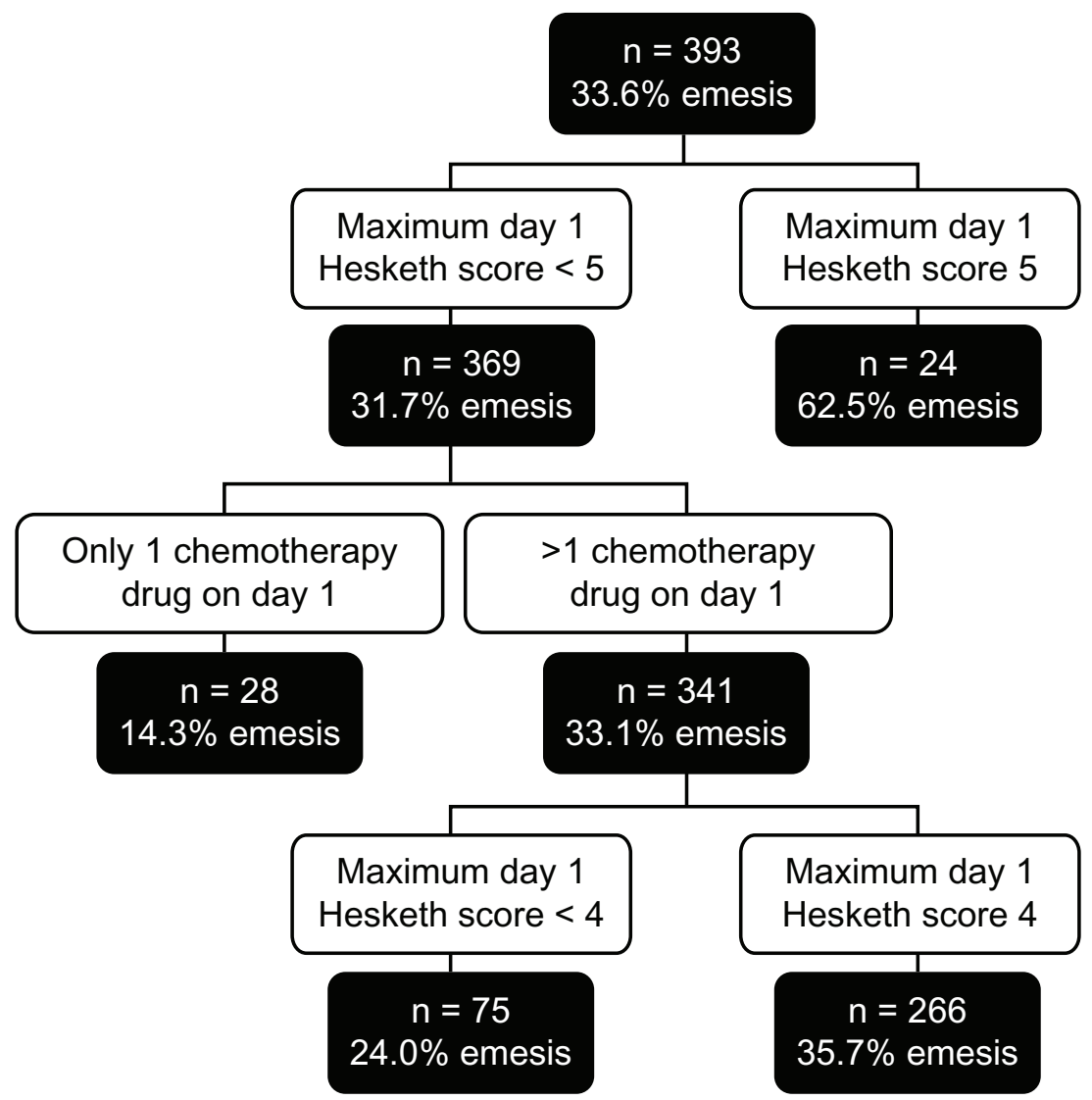

Figure 3 Classification tree for Hesketh scores,' showing that the best binary predictor of emetogenicity over the period at risk was the most emetogenic agent given on day $\mathrm{I}$. 


\section{Discussion and conclusion}

We retrospectively analyzed data from 393 patients receiving 3 days of their first cycle of a new multiday, moderately or highly emetogenic chemotherapeutic regimen in a phase III trial of GTDS versus oral granisetron. ${ }^{7}$ The results of our analyses indicate that Hesketh scores that were previously established to classify the emetogenicity of individual chemotherapeutic agents and combinations are also applicable to multiday, multiagent regimens in patients receiving prophylactic antiemetics. ${ }^{1}$ Although the simple maximum of the three daily Hesketh scores is predictive of emesis, a better predictor was found using logistic regression and classification tree techniques.

The Hesketh score on day 1 is a predictor of the emetogenicity of multiday chemotherapeutic regimens, and the presence of an individual chemotherapeutic agent with the highest Hesketh score (5) on day 1 is the greatest binary variable predictor of emesis with multiagent regimens. These findings may help guide the clinical development and use of prophylactic antiemetic regimens for preventing CINV resulting from multiday moderately or highly emetogenic regimens.

One limitation of this study is that it was retrospective and included only regimens for which the Hesketh score was at least 3. Another limitation is that the use of results from earlier studies in which patients did not receive antiemetics is not necessarily appropriate. The use of corticosteroids as rescue medication could have been a confounding factor, but the number of evaluable patients receiving rescue medication was relatively small, so no conclusions can be made about the influence of concomitant rescue medications on this analysis.

Nevertheless, we have demonstrated that the Hesketh scores as defined can provide an indication of the emetogenicity of multiday chemotherapy regimens, even in the presence of antiemetics. This study included a wide range of individual chemotherapeutic agents given in various combinations and, in this sense, the results can be thought of as generalizable.

\section{Acknowledgments}

This study was funded by ProStrakan Pharmaceuticals, Ltd, Galashiels, UK. Medical writing, editing, and graphic assistance was provided by Peloton Advantage, LLC, and was funded by ProStrakan Pharmaceuticals, Ltd. The authors thank Yvonne E Yarker, PhD, CMPP, of Peloton Advantage for providing drafts and editorial assistance during the development of the manuscript.

\section{Disclosure}

Dr Boccia reports no conflict of interest. Drs Clark and Howell are employees of ProStrakan Pharmaceuticals.

\section{References}

1. Hesketh PJ, Kris MG, Grunberg SM, et al. Proposal for classifying the acute emetogenicity of cancer chemotherapy. J Clin Oncol. 1997;15(1): 103-109.

2. Roila F, Herrstedt J, Aapro M, et al. Guideline update for MASCC and ESMO in the prevention of chemotherapy- and radiotherapy-induced nausea and vomiting: results of the Perugia consensus conference. Ann Oncol. 2010;21 Supp1 5:v232-v243.

3. Kris MG, Hesketh PJ, Somerfield MR, et al. American Society of Clinical Oncology guideline for antiemetics in oncology: update 2006. J Clin Oncol. 2006;24(18):2932-2947.

4. Grunberg SM, Osoba D, Hesketh PJ, et al. Evaluation of new antiemetic agents and definition of antineoplastic agent emetogenicity - an update. Support Care Cancer. 2005;13(2):80-84.

5. NCCN Clinical Practice Guidelines in Oncology: Antiemesis [version 1.2012]. National Comprehensive Cancer Network. Available at: http:// www.nccn.org/professionals/physician_gls/pdf/antiemesis.pdf. Accessed August 8, 2011.

6. Howell J, Smeets J, Drenth HJ, Gill D. Pharmacokinetics of a granisetron transdermal system for the treatment of chemotherapy-induced nausea and vomiting. J Oncol Pharm Pract. 2009;15(4):223-231.

7. Boccia RV, Gordan LN, Clark G, et al. Efficacy and tolerability of transdermal granisetron for the control of chemotherapy-induced nausea and vomiting associated with moderately and highly emetogenic multi-day chemotherapy: a randomized, double-blind, phase III study. Support Care Cancer. 2011;19(10):1609-1617.
Cancer Management and Research

\section{Publish your work in this journal}

Cancer Management and Research is an international, peer-reviewed open access journal focusing on cancer research and the optimal use of preventative and integrated treatment interventions to achieve improved outcomes, enhanced survival and quality of life for the cancer patient. The journal welcomes original research, clinical \& epidemiological

\section{Dovepress}

studies, reviews \& evaluations, guidelines, expert opinion \& commentary, case reports \& extended reports. The manuscript management system is completely online and includes a very quick and fair peerreview system, which is all easy to use. Visit http://www.dovepress.com/ testimonials.php to read real quotes from published authors. 